\title{
GRAPH-BASED MULTI-SCALE ANALYSIS OF PLATES AND RODS IN HUMAN TRABECULAR BONE
}

\author{
Jérôme Dias*, Sébastien Valette, Julien Dardenne, Rémy Prost, Françoise Peyrin \\ CREATIS; CNRS UMR5220; Inserm U630; INSA-Lyon; Université de Lyon, France
}

\begin{abstract}
Trabecular bone is made of a complex network of plate and rod structures, the proportion of which evolves with age or disease. Thus the identification of trabecular plates and rods is important in understanding bone fragility. We propose a novel approach based on 3D multi-scale adjacency graph analysis of high resolution 3D tomographic images of bone structures. The purpose of this new method is to classify each voxel of the 3D images in two classes : plate and rod voxels. We show that the use of a multi-scale framework is very efficient at detecting rods with different sizes. We present applications of our method to both synthetic images and experimental bone synchrotron radiation micro-CT images.
\end{abstract}

Index Terms - trabecular bone, multi-scale, clustering

\section{INTRODUCTION}

Research in the field of bone disease makes extensive use of micro$\mathrm{CT}$ to explore bone micro-architecture since micro-CT is a nondestructive means to obtain very accurate $3 \mathrm{D}$ images of bone samples. A number of quantitative three-dimensional morphometric parameters $[1,2]$ can be extracted from such images. However bone micro-architecture is made of a complex network of trabeculae and its topological organization is also of interest. It is typically organized as a mixture of plate and rod structures, the relative proportion of which evolves with age or disease. For instance, osteoporosis yields to a more rod-like structure. The SMI (Structure Model Index) which is supposed to vary between 0 (for plates) and 3 (for rods) has been introduced to quantify this organization. However, it is a global index, based on an ideal model of the structure involving the computation of the surface and its derivative [3].

To get more detailed information, local topological analysis methods have later been proposed, either based on skeleton $[4,5]$ or on medial axis transform [6]. These methods were exploited to obtain a decomposition of the structure in individual plates and rods which was correlated to biomechanical parameters $[7,8,9]$. These methods are based on skeletonization, which is a convenient tool to get simplified representations of 3D images preserving most topological information. In general 3D skeletons are not only union of curves, but are composed both of curves and medial surfaces. Intuitively, curves correspond to cylindrical parts within the object and medial surfaces to planar parts.

\footnotetext{
${ }^{*}$ Currently PhD Student in CEA-LETI, MINATEC, Grenoble, France.
}

In sharp contrast with previous approaches, we propose a new method, to locally identify plates and rods, based on multiscale Adjacency Graph $(A G)$. The first step is the segmentation of the bone regions (black in figure 1a) from the soft tissues background. The second step of the proposed algorithm is a clustering of the bone voxels. We randomly distribute clusters inside the bone region and then optimize the clustering using a Centroidal Voronoi Diagram $(C V D)$ approach [10]. The barycenter of each cell is used as a node, and an $A G$ is constructed based on the adjacency between the clusters. Afterwards, we analyze the cycles in the $A G$ to classify each edge as members of a rod or plate region. This approach is embedded into a multi-scale framework so as to reduce the algorithm sensitivity to different rods sizes. The efficiency of our approach is shown on experimental bone trabecular structures acquired in synchrotron radiation micro-CT and compared to medial axis based method [6, 11].

\section{DEFINITIONS}

Here we give some definitions used in this paper. Let the entire domain made with voxels be $\Omega$. We assume that $\Omega$ is the union of studying object domain $\Omega_{O}$ (In black figure 1a) and the external domain $\Omega_{E}$ (In white figure 1a):

$$
\Omega=\Omega_{O} \cup \Omega_{E}
$$

For clarity purposes, we will explain our approach in two dimensions. For a given image (resp. volume) $\Omega$, we associate an $A G$ obtained with a clustering of $\Omega_{O}[10]$.

\section{OUR APPROACH}

Our novel approach is based on a clustering method and processes directly the bone voxels $\Omega_{O}$.

The first step consists in creating a clustering of the bone voxels, by constructing an Approximated Centroidal Voronoi Diagram (ACVD method) in $\Omega_{O}$. The second step consists in building the corresponding $A G$ of the generated clustering. The third step is an identification of edges on which no cycle of length 3 (a triangular loop) can be found. This step allows us to determine the rod-like edges on the $A G$. Finally, we compute an edge clustering which maps each voxel of $\Omega_{O}$ to the nearest edge of the corresponding $A G$. This step is used to create the final map describing rod-like and plate-like regions. 


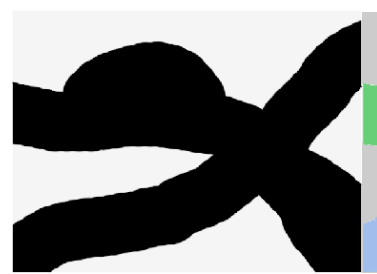

(a)

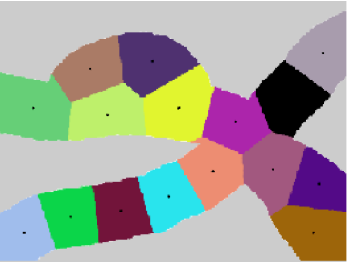

(b)

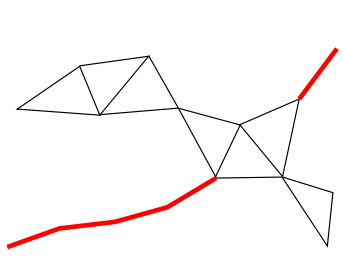

(c)

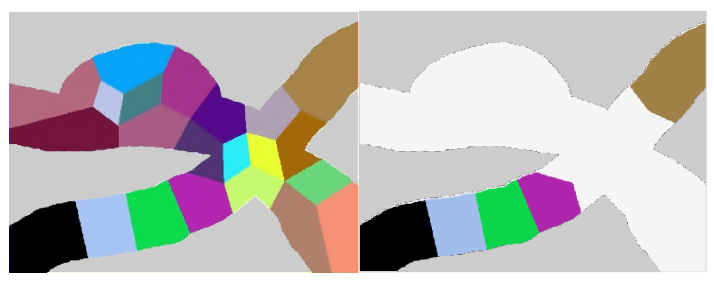

(d) (e)

Fig. 1: Our approach on $2 D$ example : Input image (1a). We construct an ACVD of the input object $\Omega_{O}(1 \mathrm{~b})$. The clustering is used to generate the $A G$ and then detect edges rod-like (colored in red in 1c). Using the $A G$, we construct the edge clustering (1d) which is finally used to identify voxels associated to rod-like edges (1e).

Figure 1 shows the main steps in our algorithm on a 2D example: the input image is shown in figure 1a. We construct an $A C V D$ of the input object $\Omega_{O}$ figure $1 \mathrm{~b}$. With this clustering, the adjacency sites of the $A C V D$ are connected by edges to form the $A G$, figure $1 \mathrm{c}$. Then we determine rod-like edges on the $A G$. Finally we identify voxels on rods figure 1e, by using the associated edge clustering figure $1 \mathrm{~d}$ and rod-like edges (red colored in figure 1c).

\subsection{Trabecular Bone Clustering}

The first step of our approach, represented figure $1 \mathrm{~b}$, is the splitting of the input object $\Omega_{O}$ into different regions $C_{i}$ by means of clustering, in spirit with $C V D$ approaches, where each region is defined by a site $z_{i}$ which is also the barycenter of his associated cell $C_{i}$ :

$$
z_{i}=\frac{\int_{C_{i}} x \cdot \rho(x) d x}{\int_{C_{i}} \rho(x) d x}
$$

Where $x$ is a point inside $\Omega_{O}$ and $\rho(x)$ a given density function. In this paper, we choose $\rho(x)=1$ everywhere, for a uniform clustering. It is known that $C V D s$ minimize the following energy term [10] :

$$
E_{V}=\sum_{i=1}^{n}\left(\sum_{p_{j} \in C_{i}} \int_{p_{j}} \rho(x)\left\|x-z_{i}\right\|^{2} d x\right)
$$

We minimize $E_{V}$, i.e. we aim at maximizing the cells compactness, in spirit with $[10,12]$. Intuitively, this approach leads to the creation of compact clusters with similar size, hence providing a solution to the sphere packing problem.

\subsection{Adjacency Graph Construction}

The $A G$ Construction consists in connecting neighbor cluster sites by an edge. In figure 1c, we show the $A G$ associated to its clustering in figure $1 \mathrm{~b}$. The voxels outside the object to be analyzed do not contribute to the $A G$. The resulting graph is then a simplified representation of the object, suitable for a fast and efficient topological analysis.

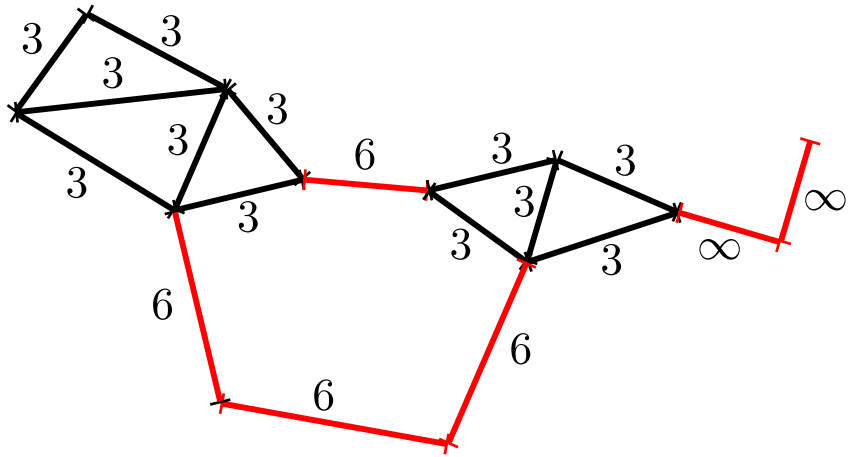

Fig. 2: Our approach on 2D graph : each edge $e$ is given a score $S_{e}$ equal to the length of the shortest cycle containing $e$. Rod-like edges (for which $S_{e}>3$ ) are colored in red.

\subsection{Rod-like edges identification}

The detection of rod-like regions depends on a rod-like edge identification step performed on the corresponding AG. We consider that non-rod edges will be plate-like edges. We use an algorithm to find edges with high likelihood of being in a rod of the bone structure. Each edge $e$ is given a score $S_{e}$ equal to the length of the shortest cycle containing $e$. Note that for consistency reasons, when an edge $e$ is not inside any cycle, we set $S_{e}=\infty$. Afterwards, a given edge $e$ is classified as rod-like if $S_{e}>3$, otherwise it is classified as platelike (fig 2). Finally, instead of computing all the cycles present in the AG which could be time consuming, we classify an edge as rod-like edge if its vertices endpoints $v_{1}$ and $v_{2}$ have no common neighbor vertex. This test is strictly equivalent to verifying that $S_{e}>3$.

\section{MULTI-SCALE ANALYSIS}

To perform our detection algorithm, one has to choose the number of regions created during the clustering. This number is very critical, as choosing a high number of clusters (little clusters size) will result in few detected rods, and a low number of clusters (large clusters size) will likely result in a lot of rods. To illustrate this problem, figure 3 shows the result of our approach on the same object, for different number of clusters. The chosen number of clusters are 33, 27 and 24: the resulting number of detected rod-like edges is respectively 0,1 and 4 . Then, instead of trying to determine the optimal num- 


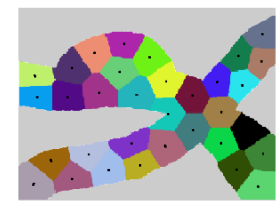

(a) Nb Clusters: 33

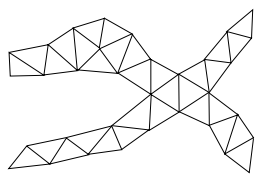

(d) rod-like edges: 0

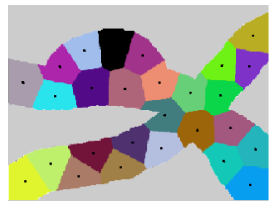

(b) Nb Clusters: 27

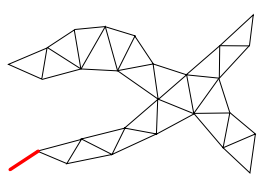

(e) rod-like edges: 1

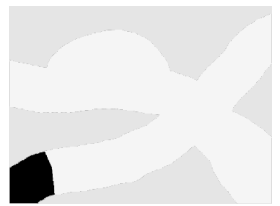

(h)

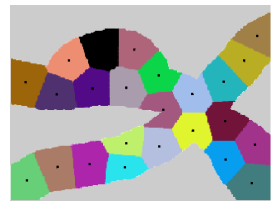

(c) Nb Clusters: 24

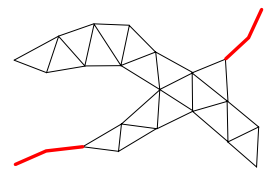

(f) rod-like edges: 4

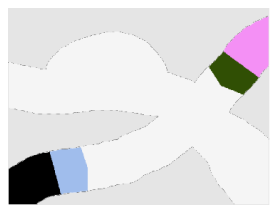

(i)
Fig. 3: MultiScale clustering on 2D example

ber of clusters, we choose to perform our algorithm in a multi-scale fashion. We first start with an arbitrary high number of clusters (typically the number of voxels in $\Omega_{E}$ divided by 50 ) and incrementally decrease this number until the clusters size are larger than the maximum rod size in bone structures, which is well documented (100 to $200 \mu \mathrm{m}$ ). The decrease is made by applying a fixed growing factor to the clusters size for each incremental step. This modification has two main advantages: we do not longer need to choose the number of clusters and we can detect small rod-like regions as well as large ones. The third row of figure 3 represent voxels associated to each rod-like edges, by using an edge clustering.

\section{GEOMETRIC FILTER}

Multi-scale analysis is a good means of avoiding to fix the number of clusters in our approach, but it brings an other problem: the chances of wrongly classifying a region as rod-like gradually increase with the clusters sizes. To overcome this difficulty, when an edge $e$ is labeled as rod-like by the AG analysis algorithm, the related voxels should verify a geometrical criterion to be effectively classified as rod-like. For a given edge, we define the sphericity criterion $\Gamma_{e}$ as:

$$
\Gamma_{e}=36 \cdot \pi \cdot \frac{V_{e}^{2}}{A_{e}^{3}}
$$

Where $V_{e}$ and $A_{e}$ are respectively the estimated volume and area of the region belonging to $e$ [13]. $V_{e}$ and $A_{e}$ are estimated by computing the inertia tensor of the set of voxels which closest edge is $e$. For a perfectly spherical region, $\Gamma_{e}=1$. In all our experiments, we discarded any edge for which $\Gamma_{e}<0.9$.

\section{RESULTS}

We have tested our algorithm and the medial axis approach [6] with a synthetic volume composed of several plates and cylinders, and both algorithms gave similar good results.

Figure 4 compares results obtained with our approach and with the medial axis approach $[6,11]$ on two 3D trabecular bone structures (A and B) imaged by synchrotron radiation micro-CT with a voxel size equal to $10 \mu \mathrm{m}$. Due the high contrast and signal to noise ratio, bone was easily segmented from background by simple thresholding.

For each $200^{3}$ voxels volume, the computation time for our algorithm is in order of several minutes, the vast majority being spent on computing clusterings. Detected rod-like regions are shown in black, while the remaining plate-like regions are transparent. For each volume, we have computed the rod/plate ratio $\tau=\frac{V r}{V p}$, where $V r$ and $V p$ are respectively the plate-like and rod-like volumes. This criterion provides an efficient way of characterizing osteoporosis. Note that the computed ratio $\tau$ are consistent with respect to visual analysis. Effectively, the volume $B$ contain more plates than rods elements, compare to the volume $A$, what explain its lower $\tau$ value. With our approach and the medial axis approach [6], we retrieve efficiently the real $\tau$ value of different phantom composed of several plates and cylinders. But we can remark that the new classification obtained on figure $4 \mathrm{~b}$, which is based on regions is more regular than that obtained on figure $4 \mathrm{c}$ based on voxels [6].

\section{CONCLUSION}

We have proposed a novel approach to identify rods and plates in 3D micro-CT images of trabecular bone which alleviates conventional noise problems encountered with skeletonization. The multi-scale scheme introduced provides robustness to the method. Our algorithm is driven by only one parameter. Application to experimental bone micro-CT images shows that the method performs well. In future works, the method will be applied to a large data set of osteoportoc and osteoarthrosic samples. The availability of this new method will open many further applications in the field of bone research for the understanding of bone fragility diseases.

\section{Acknowledgements}

This work was supported in part by the Région Rhône Alpes via both the Simed project of the ISLE research cluster and the cluster 11 HVN. This work is in the scope of the scientific topics of the PRCGDR ISIS and STIC Santé research groups of the French National Center for Scientific Research (CNRS). Our implementation is based on the Visualization ToolKit (www.vtk.org). 


\section{REFERENCES}

[1] T. Hilderbrand and P. Rüegsegger, "A new method for the model-independent assessment of thickness in threedimensional images," Journal of microscopy, vol. 185, no. 1, pp. 67-75, 1997.

[2] E. Martin-Badosa, A. Elmoutaouakkil, S. Nuzzo, D. Aamblard, L. Vico, and F. Peyrin, "A method for the automatic characterization of bone architecture in $3 \mathrm{~d}$ mice microtomographic images," Computerized Medical Imaging and Graphics, vol. 27, no. 6, pp. 447-458, 2003.

[3] T. Hildebrand and P. Rüegsigger, "Quantification of bone microarchitecture with the structure model index," Computer Methods in Biomechanics and Biomedical Engineering, vol. 1, no. 1, pp. 15 - 23, 1997.

[4] P.K. Saha, B.R. Gomberg, and F.W. Wehrli, "Threedimensional digital topological characterization of cancellous bone architecture," International Journal of Imaging Systems and Technology, vol. 11, no. 1, pp. 81-90, 2000.

[5] B.R. Gomberg, P.K. Saha, H.K. Song, S.N. Hwang, and F.W. Wehrli, "Topological analysis of trabecular bone $\mathrm{mr}$ images," IEEE Transactions on Medical Imaging, vol. 19, no. 3, 2000.

[6] A. Bonnassie, F. Peyrin, and D. Attali, "A new method for analyzing local shape in three-dimensional images based on medial axis transformation," IEEE Trans Sys Man Cyber, vol. 44, no. 4, pp. 700-705, 2003.

[7] M. Stauber and R. Müller, "Volumetric spatial decomposition of trabecular bone into rods and plates-a new method for local bone morphometry," Bone, vol. 38, no. 4, pp. 475-484, April 2006.

[8] M. Stauber, L. Rapillard, G.H. Van Lenthe, P. Zysset, and R. Muller, "Importance of individual rods and plates in the assesment of bone quality and their contribution to bone stiffness," J Bone Miner Res., vol. 21, no. 4, pp. 586-595, April 2006.

[9] X.S. Liu, P. Sajda, P.K. Saha, F.W. Wehrli, G. Bevill, T.M. Keaveny, and X.E. Guo, "Complete volumetric decomposition of individual trabecular plates and rods and its morphological correlations with anisotropic elastic moduli in human trabecular bone," J Bone Miner Res., vol. 23, no. 2, pp. 223-35, 2008.

[10] S. Valette and J.M. Chassery, "Approximated centroidal voronoi diagrams for uniform polygonal mesh coarsening," Computer Graphics Forum (Eurographics 2004 proceedings), vol. 23, no. 3, pp. 381-389, 2004.

[11] F. Peyrin, A Bonnassie, D. Attali, B. Brunet-Imbault, Chappard C., and C.L. Benhamou, "New geometric parameters for the description of three-dimensional bone structures from very high resolution microtomography images," Med. Phys., , no. 7 , in press 2010.

[12] J. Dardenne, S. Valette, N. Siauve, N. Burais, and R. Prost, "Variational tetrahedral mesh generation from discrete volume data," The Visual Computer (proceedings of CGI 2009), vol. 25, no. 5, pp. 401-410, 2009.

[13] E. Parra-Denis, Analyse morphologique 3D de particules de forme complexe: Application aux intermétalliques dans les alliages d'aluminium, Ph.D. thesis, Université de Saint-Etienne, 2007.

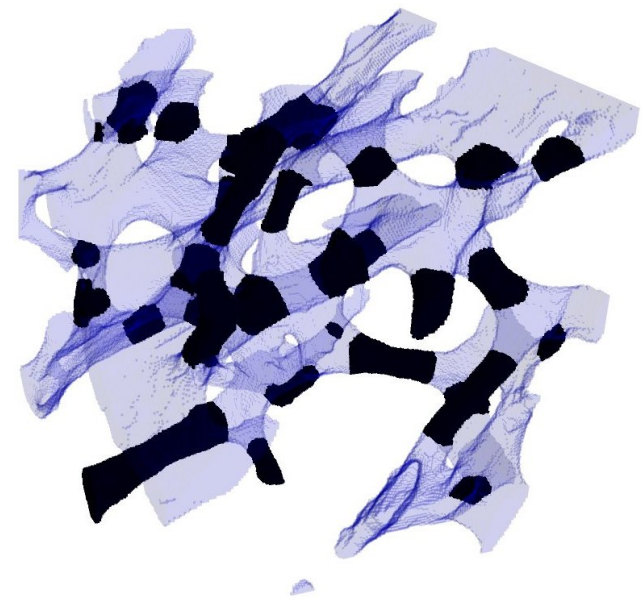

(a) Volume $A$ with our algorithm, $\tau=0.20$

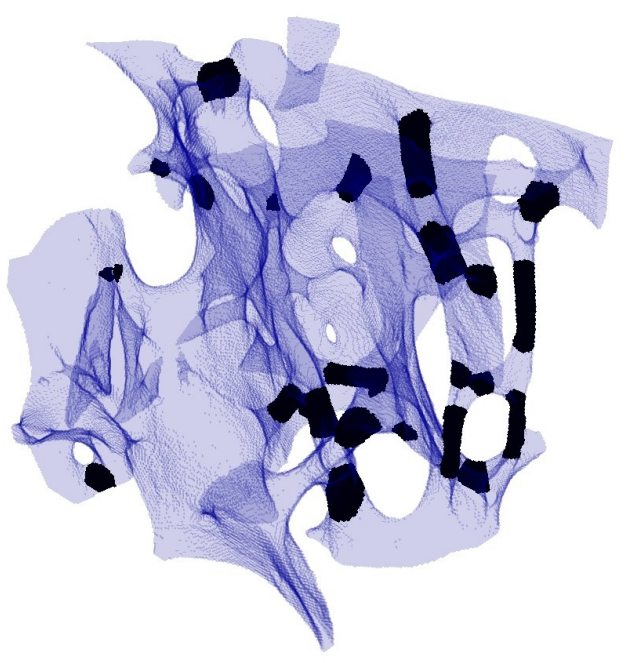

(b) Volume $B$ with our algorithm, $\tau=0.08$

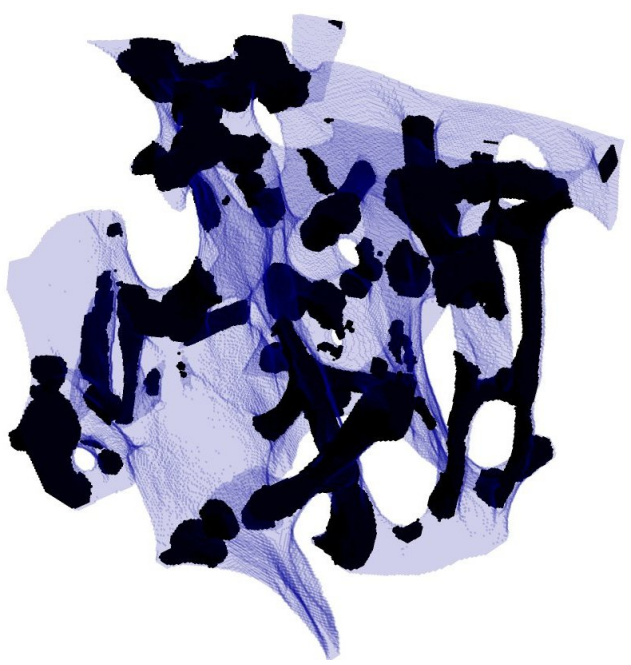

(c) Volume $B$ with [6], $\tau=0.36$

Fig. 4: Results on $200^{3}$ voxels $3 D$ trabecular bone structures, with the associated computed rod/plate ratio $\tau$. (a) and (b) are computed with our approach and (c) with [6]. 